câu hỏi được sử dụng để đánh giá hiểu biết và sự hài lòng của người bệnh có được thiết kế riêng để đánh giá cho người bệnh mổ tái tạo dây chằng chéo trước khớp gối hay cho người bệnh mổ nội soi gối nói chung và kể cả cho nhứng người bệnh có đứt dây chằng chéo trước khớp gối nhưng không mổ. Tuy vậy những kết quả bước đâuu thu thập được cũng có những ý nghĩa nhất định, giúp cho chúng ta có một cái nhìn bước đầu về tình trạng ký cam kết phẫu thuật không đồng nghĩa với việc người bệnh thực sự hiểu và chia sẻ quyết định điều trị với thầy thuốc. Kết quả nghiên cứu này sẽ là cơ sở để thực hiện các đánh giá tổng kết sâu rộng hơn sau này về vấn đề này.

\section{KẾT LUÂNN}

Người bệnh mổ tái tạo dây chằng chéo trước khớp gối được cung cấp đủ thông tin liên quan đến bệnh và phẫu thuật nhưng còn thiếu các thông tin về quá trình chăm sóc và điều trị sau mổ. Một số thông tin cần thiết được truyền tải chưa thực sự dễ hiểu. Đa số người bệnh chưa cảm thấy hài long với quá trình cung cấp thông tin cần thiết trước mổ cho phẫu thuật tái tạo dây chằng chéo trước khớp gối.

\section{TÀI LIÊU THAM KHẢO}

1. Hallock J.L., Rios R., and Handa V.L. (2017). Patient satisfaction and informed consent for surgery. Am J Obstet Gynecol, 217(2), 181.e1-181.e7.

2. Cocanour C.S. (2017). Informed consent-It's more than a signature on a piece of paper. Am J Surg, 214(6), 993-997.

3. McGaughey I. (2004). Informed consent and knee arthroscopies: an evaluation of patient understanding and satisfaction. The Knee, 11(3), 237-242.

4. Chapter 2: opinions on consent, communication \& decision making. In: American Medical Association Code of Medical Ethics; 2016:1e2. https://www. ama-assn.org/delivering-care/ama-code-medicalethics. Accessed April 13, 2017.

5. Hutson M.M. and Blaha J.D. (1991). Patients' recall of preoperative instruction for informed consent for an operation. J Bone Joint Surg Am, 73(2), 160-162.

6. Rossi M.J., Guttmann D., MacLennan M.J., et al. (2005). Video Informed Consent Improves Knee Arthroscopy Patient Comprehension. Arthrosc J Arthrosc Relat Surg, 21(6), 739-743.

7. Yin B., Goldsmith L., and Gambardella $R$. (2015). Web-Based Education Prior to Knee Arthroscopy Enhances Informed Consent and Patient Knowledge Recall: A Prospective, Randomized Controlled Study. J Bone Jt Surg, 97(12), 964-971.

8. Cornoiu A., Beischer A.D., Donnan L., et al. (2011). Multimedia patient education to assist the informed consent process for knee arthroscopy: Multimedia patient education for surgery. ANZ J Surg, 81(3), 176-180.

\title{
XÂY DỰNG THANG ĐO MỨC Độ CHẤP NHẬN TIÊM CHỦNG VẮC-XIN PHÒNG NGỪA COVID-19 TRÊN ĐỐI TƯợNG SINH VIÊN
}

\section{TÓM TẮT}

Vắc-xin là giải pháp quan trọng nhất để ngăn chặn đại dịch COVID-19 lây lan nhanh chóng. Hiện nay trền thế giới nhiều nghiên cứu đánh giá mức đô chấp nhân tiêm chủng vắc-xin phòng ngửa COVID-19 trên đối tượng sinh viên tuy nhiên chưa có nghiên cứu nào tương tự được thực hiện trong bối cảnh tại Việt Nam. Đề tài được thực hiên nhằm muc đích xây dựng thang đo khảo sát mức độ chấp nhận tiêm chủng vắc-xin ngửa COVID-19 của sinh viên. Đ̇ề tài sử dung phương pháp nghiên cứu định tính (tổng quan lý thuyết nhằm xây dựng thang đo ban đâu và thảo luận nhóm tập trung để xây dựng thang đo sơ bộ) và định lượng (bao gồm kiểm định Cronbach alpha và phân tích EFA) để hiệu chỉnh thang đo sơ bộ, từ đó đánh giá được độ tin cậy và giá trị của thang đo và hoàn thiện thang đo

*Đai hoc Y Dước Thành phố Hồ Chí Minh

Chịu trách nhiệm chính: Nguyễn Thị Thu Thủy

Email: nguyenthuthuy@ump.edu.vn

Ngày nhận bài: 7.6.2021

Ngày phản biên khoa học: 2.8.2021

Ngày duyệt bài: 9.8.2021 chính thức. Đề tài đã xây dưng thang đo mức đô chấp nhận tiêm chủng vắc-xin trển đối tượng sinh viển bao gồm 5 nhân tố tiền đề tâm lý tiêm chủng (sự tin tưởng, sự cân nhắc, trách nhiệm cộng đồng, sự tự mãn và hạn chế) với 15 biến quan sát. Thang đo đạt độ tin cậy với hệ số Cronbach's alpha của các nhân tố đều lớn hơn 0,7 và tương quan biến tổng các biến quan sát đều lớn hơn 0,3 . Phân tích EFA cho kết quả đạt yêu câu một thang đo tốt.

Tư khóa: mức độ chấp nhận vắc-xin, do dự tiêm chủng, COVID-19.

\section{SUMMARY \\ DEVELOPING SCALE OF COVID-19} VACCINE ACCEPTANCE AMONG STUDENTS

Vaccine is the most important solution to prevent the rapidly spreading COVID-19 pandemic. Currently, many studies have assessed the acceptability of vaccination against COVID-19 among students in the world but none has been conducted in the context of Vietnam. This study aimed to build a scale to assess the acceptance of COVID-19 vaccination among students. The research used qualitative methods (theoretical overview to build the initial scale and 
focus group discussions to build a preliminary scale) and quantitative methods (Cronbach-alpha test and EFA analysis) to calibrate the preliminary scale, thereby assessing the reliability and validation of the scale and completing the official one. The resulted COVID-19 vaccine acceptance scale was built including 5 psychological antecedents of vaccination: confidence, calculation, collective responsibility, complacency, constraints with 15 variables. The scale was reliable with Cronbach's alpha coefficients of all factors greater than 0.7 and the corrected item - total correlation coefficients were all greater than 0.3. EFA analysis gave satisfactory results of a good scale.

Keyword: vaccine acceptance, vaccine hesitancy, COVID-19.

\section{I. ĐĂT VẤN ĐỀ}

Kể từ khi xuất hiên vào cuối năm 2019, dịch COVID-19 đã và vẫn đang là mối quan tâm hàng đầu của thế giới bởi những ảnh hưởng nghiêm trọng của dịch bệnh đến sức khỏe và đời sống xã hội toàn cầu. Trong số các biện pháp chống lại đại dịch, tiêm chủng là một trong những biện pháp phòng ngừa hiệu quả nhất về chi phí [5]. Sau những nố lực không ngừng trong việc nghiên cứu và thử nghiệm vaccine phòng ngừa COVID-19 của các chuyên gia, tổ chức y tế, công ty dược phẩm, tính đến đâu tháng $5 / 2021$, it nhất 13 loại vaccine khác nhau trên 4 nền tảng đã được triển khai ở nhiều quốc gia trên thế giới [8]. Tiêm phòng có thể rất quan trọng để han chế sự lây lan của vi rút SARS-CoV-2 gây ra đại dịch COVID-19, nhưng khả năng miễn dịch cộng đồng chỉ có thể được thực hiện với tỷ lệ tiêm chủng cao. Sự chần chừ tiêm chủng vắc-xin phòng ngừa COVID-19 đang là một trở ngại lớn trong các nỗ lực kiểm soát đại dịch, với các bằng chứng ngày càng tăng về tính chất phổ biến của do dự tiêm chủng vắc-xin phòng ngừa COVID-19 trên các khu vực, quốc gia và tầng lớp xã hội khác nhau [2]. Hiện nay trên thế giới đã có những nghiên cứu đánh giá mức độ chấp nhận tiêm chủng vắc-xin phòng ngừa COVID-19 trền đối tượng sinh viên y tế - những nhân viên y tế tương lai sẽ tham gia lực lượng nòng cốt trong công tác phòng chống COVID-19 và ghi nhận tî̉ lệ do dự tiêm chủng vắc-xin COVID-19 khá quan ngại và cũng như các yếu tố liên quan đến hành vi tiêm chủng $[4,7]$. Tuy nhiên tại Việt Nam, thang đo đánh giá các nhân tố ảnh hưởng đến mức độ chấp nhận hay do dự tiêm chủng của sinh viển vẫn chưa được nghiển cứu đầy đủ. Vì vậy nghiên cứu "Xây dựng thang đo mức độ chấp nhận tiêm chủng vắc-xin phòng ngừa COVID-19 trên đối tượng sinh viên" được thực hiện.

\section{II. ĐỐI TƯƠNG VÀ PHƯƠNG PHÁP NGHIÊN CỨU}

Đối tượng nghiên cứu: Sinh viên khoa Dược - Đại học Y Dược

Thiết kế nghiên cứu. Nghiên cứu mô tả cắt ngang kết hợp giữa phân tích định tính và định lượng với các bước tiến hành nghiên cứu được trình bày trong hình 1.

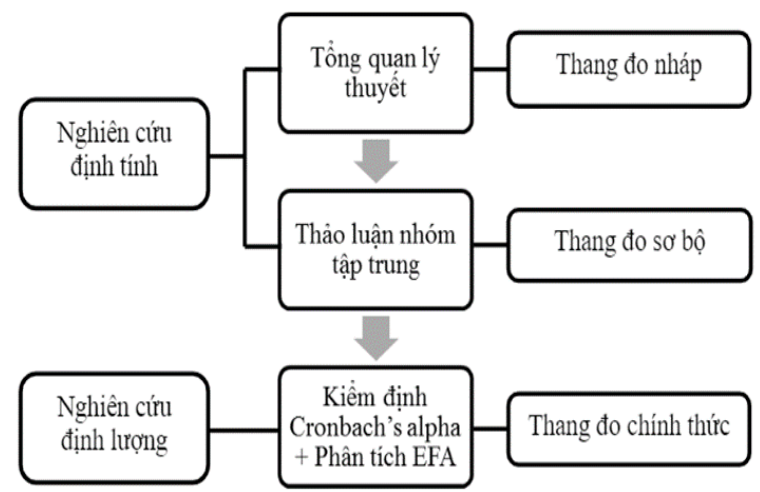

Hình 1. Các bước tiến hành nghiên cứu II. ĐỐI TƯỢNG VÀ PHƯƠNG PHÁP NGHIÊN CỨU

Nghiên cứu định tính. Thang đo ban đầu được xây dựng dựa trên tổng quan lý thuyết các nghiên cứu có liên quan và dịch thuật sang ngôn ngữ tiếng Việt, hiệu chỉnh nội dung các phát biểu cho phù hợp với đối tượng sinh viên. Sau đó tiến hành thảo luân nhóm tập trung với nhóm nhỏ 15 sinh viên ngấu nhiên từ 5 lớp chính quy tại Khoa Dược ĐHYD TP HCM (mối lớp 3 sinh viên) để kiểm tra lại nội dung từng câu hỏi, thêm hoặc bớt biến quan sát và đánh giá sơ bộ sự phù hợp của mỗi biến quan sát.

Nghiên cứu định lượng. Mô tả cắt ngang với bảng khảo sát sơ bồ gồm các câu hỏi đo lường được trình bày dưới dang thang đo 5 điểm Likert với mức độ đồng ý tăng dần từ 1 (Hoàn toàn không đồng ý) đến 5 (Hoàn toàn đồng ý). Đô tin cây và độ giá trị của thang đo được kiểm địh bằng hê số Cronbach's alpha và phân tích nhân tố EFA. Thang đo đạt độ tin cậy khi giá trị hệ số Cronbach's $a \geq 0,6$ và các giá trị tương quan biến - tổng của các biến quan sát $\geq 0,3$. Phân tích EFA được thực hiện để xác định số lượng nhân tố và cấu trúc đo lường của các biến quan sát, đồng thời kiểm định tính phù hợp của biến quan sát với nhân tố mà nó đo lường. Phân tích EFA bao gồm các bước: loại các biên có hệ số EFA nhỏ, kiểm tra nhân tố trích được, kiểm tra phương sai trích được.

\section{Mẫu nghiên cứu}

Cỡ mẫu: - Đối với phân tích nhân tố khám phá EFA, dựa theo nghiên cứu của Hair, 
Anderson, Tatham và Black (1998) [3] kích thước mẫu tối thiểu gấp 5 lần tổng số biến quan sát. Mô hình nghiên cứu có 15 biến quan sát, do đó cỡ mẫu tối thiểu là 75 sinh viên. Sinh viên được lựa chọn ngẫu nhiên từ 5 lớp chính quy của Khoa Dược Đại học Y Dược TPHCM theo tiêu chí lựa chọn và loại trừ. Sinh viên đang theo học hệ chính quy tại khoa Dược -Đại học Y Dược TPHCM trong thời gian nghiên cứu, tuyến,

- Có khả năng tiếp cận với bảng câu hỏi trực

- Đồng ý tham gia nghiên cứu.

Tiêu chí loại trừ:

- Sinh viên có từ 2 phiếu khảo sát trở lên: lây phiếu đầu tiên và loại phiếu lặp

- Phiếu khảo sát vi phạm câu hỏi record (câu hỏi nghịch đảo)

- Sinh viên đã được tiêm chủng vắc-xin phòng ngừa COVID-19

Phương pháp lấy mẫu: nghiên cứu tiến hành lấy mẫu toàn bộ trong thời gian tháng 4$6 / 2021$. Phiếu khảo sát được gứi tới tài khoản thư điện tử cá nhân của sinh viên do nhà trường cung cấp và quản lý.

Phương pháp thống kê và xử lý số liệu: Dữ liệu của đề tài được xử lý và thống kê bắng phần mềm Microsoft Excel 2010, IBM SPSS
Statistics 20.0. Độ tin cậy 95\% được sử dụng để kiểm tra các giả thiết thống kê.

\section{KẾT QUẢ NGHIÊN CỨU}

Xây dựng thang đo nháp. Cơ quan tư vấn của WHO (Nhóm Cố vấn Chiến lược của các Chuyên gia về Tiêm chủng - SAGE) đề ra mô hình $3 C$ các nhân tố ảnh hưởng đến mức độ chấp nhận hay do dự tiêm chủng bao gồm: sự tin tưởng (confidence), sự tự mãn (complacency) và sự thuận tiện (convenience) [6]. Dựa trên các đánh giá tài liệu và cân nhắc lý thuyết, Betsch và cộng sự đã mở rộng mô hình $3 C$ thành mô hình $5 \mathrm{C}$ bằng cách tích hợp sự cân nhắc (calculation) và trách nhiệm cộng đồng (collective responsibility) [1]. Thang đo $5 \mathrm{C}$ của Betsch và cộng sự xây dựng dựa trên mô hình $5 \mathrm{C}$ khảo sát cả 5 yểu tố ảnh hưởng đến do dự tiêm chủng trong khi hầu hết các thang đo hiện có chủ yếu tập trung vào các khía cạnh liên quan đến sự tin tưởng (confidence) và chỉ đôi khi vượt ra ngoài yếu tố chính này [1],

Dựa trên nghiên cứu của Betsch và cộng sự [1], đề tài đề xuất mô hình các nhân tố ảnh hưởng đến mức độ chấp nhận tiêm chủng vắc-xin trên đối tượng sinh viên bao gồm 5 nhân tố tiền đề tâm lý tiêm chủng được trình bày trong hình 2 .

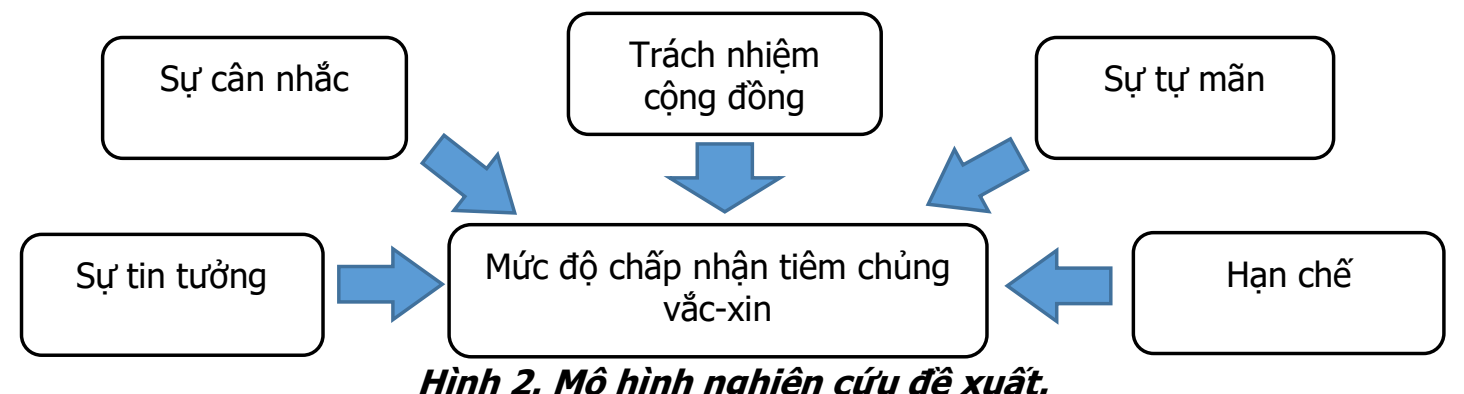

Thang đo ban đầu bao gồm 5 nhân tố: sự tin tưởng, sự cân nhắc, trách nhiệm cộng đồng, sự tự mãn, hạn chế; trong đó mô̂i nhân tố được đo bằng 3 biến quan sát; thang đo Likert 5 mức độ được sử dụng để đo lường từng biến quan sát với 1 - Hoàn toàn không đồng ý và 5 - Hoàn toàn đồng ý. Các biến quan sát dựa vào thang đo của Betsch và cộng sự [1] với nội dung các phát biểu được Việt hóa bởi 2 người nghiên cứu độc lập. Sau 3 lần dịch đi dịch lại Anh-Việt-Anh, nội dung các phát biểu được trình bày trong bảng 1 .

Bảng 1. Nội dung phát biểu trong thang đo

\begin{tabular}{|c|c|c|c|}
\hline $\begin{array}{l}\text { Thứ } \\
\text { tự }\end{array}$ & $\begin{array}{l}\text { Thang đo } \\
\text { nhân tố }\end{array}$ & $\begin{array}{c}\text { Ký } \\
\text { hiệu }\end{array}$ & Biến quan sát \\
\hline \multirow{3}{*}{1} & \multirow{3}{*}{$\begin{array}{l}\text { Sự tin } \\
\text { tưởng }\end{array}$} & STT1 & Tôi hoàn toàn tin tưởng vắc-xin COVID-19 an toàn. \\
\hline & & STT2 & Vắc-xin COVID-19 có hiệu quả. \\
\hline & & STT3 & $\begin{array}{l}\text { Đối với vắc-xin COVID-19, tôi tin tưởng rằng các cơ quan công quyền } \\
\text { có quyết định vì lợi ích tốt nhất của cộng đồng. }\end{array}$ \\
\hline \multirow[t]{2}{*}{2} & \multirow[t]{2}{*}{ Hạn chế } & $\mathrm{HC} 1$ & $\begin{array}{l}\text { Các áp lực cuộc sống hàng ngày ngăn cản tôi tiêm chủng vắc-xin } \\
\text { COVID-19. }\end{array}$ \\
\hline & & $\mathrm{HC2}$ & Việc tiêm chủng vắc-xin COVID-19 đối với tôi là bất tiện. \\
\hline
\end{tabular}




\begin{tabular}{|c|c|c|c|}
\hline & & HC3 & $\begin{array}{l}\text { Đến gặp bác sĩ khiến tôi cảm thấy khó chịu, điều này khiến tôi không } \\
\text { muốn đi tiêm chủng vắc-xin COVID-19. }\end{array}$ \\
\hline \multirow{3}{*}{3} & \multirow{3}{*}{ Sự tự mãn } & STM1 & $\begin{array}{c}\text { Tiêm phòng vắc-xin COVID-19 là không cần thiết vì dịch bệnh đã } \\
\text { được kiểm soát tốt. }\end{array}$ \\
\hline & & STM2 & Hệ thống miễn dịch của tôi rất mạnh, bảo vệ tôi chống lại COVID-19. \\
\hline & & STM3 & $\begin{array}{l}\text { Bệnh do COVID-19 không ảnh hưởng nghiêm trọng tới sức khỏe đến } \\
\text { mức tôi nên đi tiêm phòng. }\end{array}$ \\
\hline \multirow{3}{*}{4} & \multirow{3}{*}{$\begin{array}{l}\text { Sự cân } \\
\text { nhắc }\end{array}$} & SCN1 & $\begin{array}{l}\text { Khi nghĩ đến việc tiêm chủng vắc-xin COVID-19, tôi cân nhắc giữa lợi } \\
\text { ích và rủi ro để đưa ra quyết định tốt nhất có thể. }\end{array}$ \\
\hline & & SCN2 & Tôi xem xét kỹ lưỡng vắc-xin COVID-19 có hữu ích cho tôi hay không. \\
\hline & & SCN3 & $\begin{array}{l}\text { Điêu quan trọng đối với tôi là tôi phải hiểu đầy đủ về vắc-xin COVID- } \\
19 \text { trước khi tôi tiêm chủng. }\end{array}$ \\
\hline \multirow{3}{*}{5} & \multirow{3}{*}{$\begin{array}{c}\text { Trách } \\
\text { nhiệm } \\
\text { cộng đồng }\end{array}$} & TNCD1 & $\begin{array}{l}\text { Khi tất cả mọi người đều được tiêm chủng vắc-xin COVID-19, tôi } \\
\text { không cần phải tiêm chủng vắc-xin COVID-19. }\end{array}$ \\
\hline & & TNCD2 & $\begin{array}{l}\text { Tôi tiêm chủng vắc-xin COVID-19 vì tôi có thể bảo vệ cả những người } \\
\text { yếu hơn tôi. }\end{array}$ \\
\hline & & TNCD3 & $\begin{array}{l}\text { Tiêm chủng vắc-xin COVID-19 là một hành động tập thể để ngăn } \\
\text { chặn sự lây lan của dịch bệnh. }\end{array}$ \\
\hline
\end{tabular}

Xây dựng thang đo sơ bộ. Để hiệu chỉnh thang đo phù hợp với đối tượng sinh viển, đồng thời điều chỉnh nội dung các phát biểu và đánh giá sơ bộ mức độ phù hợp của biến quan sát với các thành phần nhân tố, đề tài tiến hành phỏng vấn nhóm nhỏ 15 sinh viên ngẫu nhiên từ 5 lớp chính quy tại Khoa Dược ĐHYD TP HCM (mỗi lớp 3 sinh viên). Sau khi thảo luận nhóm, hầu hết sinh viên đều đồng thuận các thang đo thành phần do đề tài đưa ra. Không có sự thay đổi về số lượng và tên gọi các thành phần và các biến so với mô hình nghiên cứu đề xuất. Tuy nhiên, môt số điều chỉnh về thứ tư câu hỏi và nôi dung phát biểu một số nhân tố cần được thực hiện với nội dung chi tiết được trình bày trong bảng 2 .

Bảng 2. Hiệu chỉnh thang đo sau khi thảo luận nhóm

\begin{tabular}{|c|c|c|}
\hline Nội dung & Thang đo sơ bộ & Thang đo hiệu chỉnh \\
\hline Thứ tự câu hỏi & $\begin{array}{c}\text { Xen kẽ sự tự tin - hạn chế - sự } \\
\text { tự mẫn - sự cân nhắc - trách } \\
\text { nhiệm cộng đồng }\end{array}$ & $\begin{array}{l}\text { Nhóm laai các yếu tố mang tính tích cực } \\
\text { (sự tự tin, sự cân nhắc, trách nhiệm cộng } \\
\text { đồng) và tiểu cực (hạn chế, sự tự mãn) }\end{array}$ \\
\hline $\begin{array}{l}\text { Phát biểu đối với nhân } \\
\text { tố trách nhiệm cộng } \\
\text { đồng }\end{array}$ & $\begin{array}{l}\text { Tôi tiêm chủng vắc-xin COVID- } \\
19 \text { vì tôi có thể bảo về cả } \\
\text { những người yếu hơn tôi. }\end{array}$ & $\begin{array}{c}\text { Tôi tiêm chủng vắc-xin COVID-19 vì tôi có } \\
\text { thể bảo vệ cả những người xung quanh } \\
\text { tôi. }\end{array}$ \\
\hline $\begin{array}{l}\text { Phát biểu đối với nhân } \\
\text { tố hạn chế }\end{array}$ & $\begin{array}{c}\text { Đến gă̆p bác sĩ khiến tôi cảm } \\
\text { thấy khó chịu, điều này khiến } \\
\text { tôi không muốn đi tiêm chủng } \\
\text { vắc-xin COVID-19. }\end{array}$ & $\begin{array}{l}\text { Đến gặp bác sĩ khiến tôi cảm thấy không } \\
\text { thoải mái, điêuu này khiến tôi không } \\
\text { muốn đi tiêm chủng vắc-xin COVID-19. }\end{array}$ \\
\hline
\end{tabular}

\section{Xây dựng thang đo chính thức}

Đánh giá sơ bố độ tin cậy của thang đo. Dựa trên cơ sở dữ liệu thu thập được từ 75 phiếu khảo sát đã hoàn thành trong khảo sát sơ bộ, độ tin cậy của thang đo sơ bộ được đánh giá bằng hệ số Cronbach's alpha. Kết quả kiểm định Cronbach's alpha được trình bày trong bảng 3 .

Bảng 0. Kêt quả kiểm định Cronbach's alpha thang đo sơ bộ

\begin{tabular}{|c|c|c|}
\hline Nhân tố & $\begin{array}{c}\text { Biến quan } \\
\text { sát }\end{array}$ & $\begin{array}{c}\text { Tương quan } \\
\text { biến - tổng }\end{array}$ \\
\hline \multirow{2}{*}{$\begin{array}{c}\text { Sự tin tưởng } \\
\mathrm{a}=0,793\end{array}$} & $\mathrm{STT1}$ & 0,688 \\
\cline { 2 - 3 } & $\mathrm{STT} 2$ & 0,701 \\
\cline { 2 - 3 } & $\mathrm{STT} 3$ & 0,537 \\
\hline Sự cân nhắc & $\mathrm{SCN} 1$ & 0,527 \\
\hline
\end{tabular}

\begin{tabular}{|c|c|c|}
\hline $\mathrm{a}=0,743$ & SCN2 & 0,607 \\
\cline { 2 - 3 } & SCN3 & 0,596 \\
\hline Trách nhiệm & TNCD1 & 0,330 \\
\cline { 2 - 3 } cộng đồng & TNCD2 & 0,418 \\
\cline { 2 - 3 }$=0,631$ & TNCD3 & 0,639 \\
\hline \multirow{2}{*}{ Sự tự mãn } & STM1 & 0,712 \\
\cline { 2 - 3 }$a=0,874$ & STM2 & 0,762 \\
\cline { 2 - 3 } & STM3 & 0,813 \\
\hline \multirow{2}{*}{ Hạn chễ } & HC1 & 0,724 \\
\cline { 2 - 3 }$a=0,888$ & HC2 & 0,871 \\
\cline { 2 - 3 } & HC3 & 0,754 \\
\hline
\end{tabular}

Ghi chú: STT - sự tin tưởng, SCN - sự cân nhắc, TNCD - trách nhiệm cộng đồng, STM - sự tự mãn, $\mathrm{HC}$ - hạn chế

Theo bảng 3, tương quan biến - tổng của các 
biến quan sát đều lớn hơn 0,3 với giá trị nhỏ nhất là 0,330 của biến TNCD1. Đề tài ghi nhận giá trị Cronbach's alpha ở 4 thang đo thành phẩn gồm sự tin tưởng, sự cân nhắc, sự tự mãn, hạn chế tổng thể đều ở mức tốt $(a>0,7)$. Giá trị Cronbach's alpha ở thang đo trách nhiệm cộng đồng ở mức chấp nhận được $(a>0,6)$. Như vậy, các thang đo đều đạt độ tin cậy.

Đánh giá sơ bộ tính hội tụ và phân biệt của thang đo. Để kiểm định độ giá trị của thang đo thông qua độ hội tụ và giá trị phân biệt của các biến quan sát trong thang đo, đề tài tiển hành phân tích nhân tố EFA sử dụng phương pháp trích Principal Component với phép xoay Varimax. Kết quả phân tích EFA được trình bày trong bảng 4.

Bảng 4. Kêt quả xoay nhân tố thang đo các thành phän thang đo sơ bộ.

\begin{tabular}{|c|c|c|c|c|}
\hline \multirow{2}{*}{$\begin{array}{c}\text { Biến quan } \\
\text { sát }\end{array}$} & \multicolumn{5}{|c|}{ Nhân tố } \\
\hline & 1 & 2 & 3 & 4 \\
\hline STM3 & 0,866 & & & \\
\hline STM2 & 0,852 & & & \\
\hline STM1 & 0,823 & & & \\
\hline TNCD1 & $-0,704$ & & & \\
\hline STT2 & & 0,827 & & \\
\hline STT1 & & 0,827 & & \\
\hline TNCD3 & & 0,695 & & \\
\hline TNCD2 & & 0,660 & & \\
\hline STT3 & & 0,600 & & \\
\hline HC2 & & & 0,866 & \\
\hline HC1 & & & 0,826 & \\
\hline HC3 & & & 0,792 & \\
\hline SCN2 & & & & 0,830 \\
\hline SCN1 & & & & 0,794 \\
\hline SCN3 & & & & 0,772 \\
\hline Eigenvalue & 5,141 & 2,667 & 1,713 & 1,176 \\
\hline $\begin{array}{l}\text { Phương sai } \\
\text { trích (\%) }\end{array}$ & 34,276 & 52,058 & 63,478 & 71,318 \\
\hline \multicolumn{4}{|c|}{ KMO $=\mathbf{0 , 7 5 3}(\mathbf{p = 0 , 0 0 0 )}$} \\
\hline
\end{tabular}

Theo bảng 4, đề tài ghi nhận hệ số KMO = $0,753>0,5$ như vậy phân tích nhân tố thích hợp với dữ liệu nghiền cứu. Kết quả kiểm định Barlett's là 592,022 với mức ý nghĩa sig $=0,000$ $<0,05$. Điều này chứng tỏ dứ liệu dùng để phân tích nhân tố là hoàn toàn thích hợp. Có 4 nhân tố được trích tại Eigenvalue 1,176 và tổng phương sai trích là $71,318 \%$ (> 50\%). Như vậy, số lượng nhân tố nhóm từ các biến quan sát chưa đạt kỳ vọng với thang đo đề xuất ban đâu. Các biển quan sát đều có hệ số tải $>0,5$ và đạt được giá trị hội tụ và giá trị phân biệt ngoại trừ biến TNCD1 không đạt giá trị hội tụ (hệ số tải có giá trị $-0,704<0,5)$.

Hiệu chỉnh thang đo. Dựa trên kết quả khảo sát tính giá trị của thang đo, đề tài tiến hành khảo sát thêm 15 sinh viên ngấu nhiên từ 5 lớp chính quy để xác định hướng hiệu chỉnh của phát biểu TNCD1 không đạt giá trị hội tụ với kết quả được trình bày trong bảng 5 .

Bảng 5. Hiệu chinh nội dung phát biểu thang đo trách nhiệm cộng đồng

\begin{tabular}{|c|c|c|}
\hline $\begin{array}{c}\text { Biến } \\
\text { quan sát }\end{array}$ & Phát biểu gốc & $\begin{array}{l}\text { Phát biểu hiệu } \\
\text { chỉnh }\end{array}$ \\
\hline TNCD1 & $\begin{array}{l}\text { Khi tất cả mọi } \\
\text { người đều được } \\
\text { tiêm chủng vắc- } \\
\text { xin COVID-19, tôi } \\
\text { không cần phải } \\
\text { tiêm chủng vắc- } \\
\text { xin COVID-19 }\end{array}$ & $\begin{array}{l}\text { Khi tất cả mọi } \\
\text { người đều được } \\
\text { tiêm chủng vắc-xin } \\
\text { COVID-19, tôi vẫn } \\
\text { cần phải tiêm } \\
\text { chủng vắc-xin } \\
\text { COVID-19 }\end{array}$ \\
\hline
\end{tabular}

Sau khi hiệu chỉnh, đề tài tiến hành đánh giá độ tin cậy và tính giá trị của thang đo hiệu chỉnh trền mẫu 75 sinh viên ngẫu nhiên từ 5 lởp chính quy tại Khoa Dược ĐHYD TP HCM.

Kểt quả đánh giá độ tin cậy của thang đo sau hiệu chỉnh được trình bày trong bảng 6 .

Bảng 6. Kêt quả kiểm định Cronbach's alpha thang đo hiêu chinh

\begin{tabular}{|c|c|c|}
\hline Khái niệm & $\begin{array}{c}\text { Biến quan } \\
\text { sát }\end{array}$ & $\begin{array}{l}\text { Tương quan } \\
\text { biến - tổng }\end{array}$ \\
\hline \multirow{3}{*}{$\begin{array}{l}\text { Sự tin tưởng } \\
a=0,707\end{array}$} & STT1 & 0,517 \\
\hline & STT2 & 0,595 \\
\hline & STT3 & 0,493 \\
\hline \multirow{3}{*}{$\begin{array}{c}\text { Sự cân nhắc } \\
a=0,835\end{array}$} & SCN1 & 0,740 \\
\hline & SCN2 & 0,704 \\
\hline & SCN3 & 0,669 \\
\hline \multirow{3}{*}{$\begin{array}{c}\text { Trách nhiệm } \\
\text { cộng đồng } \\
a=0,873\end{array}$} & TNCD1 & 0,763 \\
\hline & TNCD2 & 0,802 \\
\hline & TNCD3 & 0,709 \\
\hline \multirow{3}{*}{$\begin{array}{l}\text { Sự tự mãn } \\
a=0,813\end{array}$} & STM1 & 0,597 \\
\hline & STM2 & 0,725 \\
\hline & STM3 & 0,692 \\
\hline \multirow{3}{*}{$\begin{array}{c}\text { Hạn chế } \\
a=0,777\end{array}$} & $\mathrm{HC1}$ & 0,560 \\
\hline & $\mathrm{HC2}$ & 0,722 \\
\hline & $\mathrm{HC} 3$ & 0,564 \\
\hline
\end{tabular}

Theo bảng 6, tương quan biến - tống của các biến quan sát đều lớn hơn 0,3 với giá trị nhỏ nhất là 0,493 (biến STT3). Đề tài ghi nhận giá trị Cronbach's alpha ở cả 5 thang đo thành phân đều ở mức tốt $(>0,7)$. Như vậy, các thang đo đều đạt độ tin cậy. Kết quả phân tích EFA thang đo sau hiệu chỉnh được trình bày trong bảng 7 .

Bảng 7. Kêt quả xoay nhân tố thang đo các thành phần thang đo sau hiệu chỉnh.

\begin{tabular}{|c|c|c|c|c|c|}
\hline \multirow{2}{*}{$\begin{array}{c}\text { Biến quan } \\
\text { sát }\end{array}$} & $\mathbf{1}$ & $\mathbf{2}$ & $\mathbf{3}$ & $\mathbf{4}$ & $\mathbf{5}$ \\
\hline TNCD1 & 0,898 & & & & \\
\hline TNCD2 & 0,858 & & & & \\
\hline
\end{tabular}




\begin{tabular}{|c|c|c|c|c|c|}
\hline TNCD3 & 0,821 & & & & \\
\hline SCN2 & & 0,892 & & & \\
\hline SCN1 & & 0,860 & & & \\
\hline SCN3 & & 0,812 & & & \\
\hline STM2 & & & 0,855 & & \\
\hline STM3 & & & 0,801 & & \\
\hline STM1 & & & 0,791 & & \\
\hline HC2 & & & & 0,865 & \\
\hline HC1 & & & & 0,823 & \\
\hline HC3 & & & & 0,665 & \\
\hline ST12 & & & & & 0,866 \\
\hline STT1 & & & & & 0,704 \\
\hline STT3 & & & & & 0,654 \\
\hline Eigenvalue & 4,538 & 2,411 & 2,083 & 1,243 & 1,133 \\
\hline $\begin{array}{l}\text { Phương sai } \\
\text { trích (\%) }\end{array}$ & 30,256 & 46,327 & 60,216 & 68,506 & 76,058 \\
\hline \multicolumn{5}{|c|}{ KMO = 0,739 (p=0,000) } \\
\hline
\end{tabular}

Theo bảng 7, đề tài ghi nhận hệ số KMO = $0,739>0,5$ như vậy phân tích nhân tố thích hợp với dữ liệu nghiên cứu. Kết quả kiểm định Barlett's có giá trị 531,774 với mức ý nghĩa sig = $0,000<0,05$. Điều này chứng tỏ dữ liệu dùng để phân tích nhân tố là hoàn toàn thích hợp. Có 5 nhân tố được trích tại Eigenvalue 1,133 và tổng phương sai trích là 76,058\% (> 50\%). Như vậy, số lượng nhân tố nhóm từ các biến quan sát phù hợp với thang đo đề xuất ban đâu. Các biến quan sát đều có hệ số tải > 0,5 và đạt được giá trị hội tụ và giá trị phân biệt.

Xây dưng thang đo chính thức. Dựa trên kết quả đánh giá sơ bộ và hiệu chỉnh, đề tài xây dựng thang đo chính thức gồm 5 nhân tố với 15 biến quan sát. Các câu hỏi trong thang đo chính thức đước trình bày dưới dang câu hỏi 5 điểm Likert với 1-Hoàn toàn không đồng ý tới 5-Hoàn toàn đồng ý. Nội dung các biến quan sát được trình bày trong bảng 8 .

\section{Bảng 8. Biên số khảo sát của thang đo chính thức}

\begin{tabular}{|c|c|c|}
\hline Khái niệm & Biến quan sát & Kí hiệu \\
\hline \multirow{3}{*}{$\begin{array}{l}\text { Sự tin } \\
\text { tưởng }\end{array}$} & Tôi hoàn toàn tin tưởng vắc-xin COVID-19 an toàn. & STT1 \\
\hline & Vắc-xin COVID-19 có hiệu quả. & STT2 \\
\hline & $\begin{array}{l}\text { Đối với vắc-xin COVID-19, tôi tin tưởng rằng các cơ quan công quyên } \\
\text { có quyết định vì lợi ích tốt nhất của cộng đồng. }\end{array}$ & STT3 \\
\hline \multirow{3}{*}{$\begin{array}{l}\text { Sự cân } \\
\text { nhắc }\end{array}$} & $\begin{array}{l}\text { Khi nghĩ đến việc tiêm chủng vắc-xin COVID-19, tôi cân nhắc giữa lợi } \\
\text { ích và rủi ro để đưa ra quyết định tốt nhất có thể. }\end{array}$ & SCN1 \\
\hline & Tôi xem xét kỹ lương vắc-xin COVID-19 có hữu ích cho tôi hay không. & SCN2 \\
\hline & $\begin{array}{l}\text { Điều quan trọng đối với tôi là tôi phải hiểu đâyy đủ về vắc-xin COVID- } \\
19 \text { trước khi tôi tiêm chủng. }\end{array}$ & SCN3 \\
\hline \multirow{3}{*}{$\begin{array}{c}\text { Trách } \\
\text { nhiệm } \\
\text { cộng đông }\end{array}$} & $\begin{array}{l}\text { Khi tất cả mọi người đều được tiêm chủng vắc-xin COVID-19, tôi vấn } \\
\text { cânn phải tiêm chủng vắc-xin COVID-19. }\end{array}$ & TNCD1 \\
\hline & $\begin{array}{l}\text { Tôi tiêm chủng vắc-xin COVID-19 vì tôi có thể bảo vệ cả những người } \\
\text { xung quanh tôi. }\end{array}$ & TNCD2 \\
\hline & $\begin{array}{l}\text { Tiêm chủng vắc-xin COVID-19 là một hành động tập thể để ngăn chặn } \\
\text { sự lây lan của dịch bệnh. }\end{array}$ & TNCD3 \\
\hline \multirow{3}{*}{ Sự tự mãn } & $\begin{array}{l}\text { Tiêm phòng vắc-xin COVID-19 là không cân thiết vì dịch bệnh đã được } \\
\text { kiểm soát tốt. }\end{array}$ & STM1 \\
\hline & Hệ thống miến dịch của tôi rất mạnh, bảo vệ tôi chống lại COVID-19. & STM2 \\
\hline & $\begin{array}{c}\text { Bệnh do COVID-19 không ảnh hưởng nghiêm trọng tới sức khỏe đến } \\
\text { mức tôi nên đi tiêm phòng. }\end{array}$ & STM3 \\
\hline \multirow{3}{*}{ Hạn chế } & $\begin{array}{l}\text { Các áp lực cuộc sống hàng ngày ngăn cản tôi tiêm chủng vắc-xin } \\
\text { COVID-19. }\end{array}$ & $\mathrm{HC} 1$ \\
\hline & Việc tiêm chủng vắc-xin COVID-19 đối với tôi là bất tiện. & $\mathrm{HC2}$ \\
\hline & $\begin{array}{l}\text { Đến gặp bác sĩ khiến tôi cảm thấy không thoải mái, điều này khiến tôi } \\
\text { không muốn đi tiêm chủng vắc-xin COVID-19. }\end{array}$ & $\mathrm{HC} 3$ \\
\hline
\end{tabular}

\section{BÀN LUÂN}

Đây là một trong những nghiên cứu đầu tiên xây dựng thang đo khảo sát mức độ chấp nhận tiêm chủng vắc-xin ngừa COVID-19 trên đối tượng sinh viên tại Việt Nam. Thang đo khảo sát cả 5 yếu tố tiền đề tâm lý quyết định hành vi tiêm chủng vắc-xin được đề xuất bởi mô hình ban đầu. Kết quả thẩm định độ tin cậy và hiệu lực cho thấy thang đo đạt độ tin cậy cao với giá trị Cronbach's alpha ở cả 5 thang đo thành phần đều ở mức tốt, số lượng nhân tố nhóm từ các biến quan sát phù hợp và các biến quan sát đều 
đạt được giá trị hội tụ và giá trị phân biệt. Đặt ở bối cảnh hiện tại, việc tiêm chủng vắc-xin ngừa COVID-19 là giải pháp quan trọng nhất ngăn chặn dịch bệnh trong khi vẫn tồn tại một tỉ lệ do dự tiêm chủng trong dân số, nghiên cứu cung cấp công cụ cần thiết với độ tin cậy cao để đánh giá mức độ chấp nhận tiêm chủng vắc-xin COVID-19 và các yếu tố ảnh hưởng, giúp đưa ra các chiến lược hành động phù hợp, hiệu quả, nâng cao tỉ iệ tiêm chủng vắc-xin phòng ngừa COVID-19 đạt mục tiêu miển dịch cộng đồng.

Tuy nhiên, thang đo xây dựng được đánh giá đạt độ tin cậy và tính giá trị cho đối tượng sinh viên. Trong trường hợp áp dụng thang đo cho những đối tượng khác (người dân, cán bộ y tế...) cần có những điều chỉnh phù hợp và được thẩm định lại về độ tin cậy và tính giá trị. Với giới hạn về thời gian tiến hành nghiên cứu, giá trị dự báo của thang đo về mức độ chấp nhận tiêm chủng vắc-xin chưa được đánh giá. Do đó các nghiên cứu trong tương lai có thể khảo sát giá trị dự báo của thang đo để có bức tranh toàn cảnh hơn về giá trị của thang đo.

\section{KẾT LUẬN}

Thang đo đánh giá mức độ chấp nhận tiêm chủng vắc-xin COVID-19 trên đối tượng sinh viên đã được xây dựng với 5 nhân tố tiền đề tâm lý tiêm chủng bao gồm sự tin tưởng, sự cân nhắc, trách nhiệm cộng đồng, sự tự mãn và hạn chế và 15 biến quan sát. Các kết quả kiểm định cho thấy thang đo đạt độ tin cậy và độ giá trị cao. Thang đo có thể sử dụng trong tương lai để khảo sát mức độ chấp nhận tiêm chủng vắc-xin COVID-19 trên đối tượng sinh viên và các yếu tố liên quan cũng như làm cơ sở đề ra các giải pháp can thiệp phù hợp.

\section{TÀI LIÊUU THAM KHẢO}

1. Betsch C. et al. (2018), "Beyond confidence: Development of a measure assessing the $5 \mathrm{C}$ psychological antecedents of vaccination", PloS one. 13 (12), pp. e0208601.

2. Dror A. A. et al. (2020), "Vaccine hesitancy: the next challenge in the fight against COVID-19", Eur J Epidemiol. 35 (8), pp. 775-779.

3. Hair J., Anderson, R., Tatham, R. and Black, W. (1998), Multivariate data analysis, Prentice Hall, New Jersey.

4. Kanyike A. M. et al. (2021), "Acceptance of the coronavirus disease-2019 vaccine among medical students in Uganda", Tropical Medicine and Health. 49 (1), pp. 1-11.

5. Lurie N. et al. (2020), "Developing Covid-19 vaccines at pandemic speed", New England Journal of Medicine. 382 (21), pp. 1969-1973.

6. MacDonald N. E. (2015), "Vaccine hesitancy: Definition, scope and determinants", Vaccine. 33 (34), pp. 4161-4164.

7. Mascarenhas A. K. et al. (2021), "Dental students' attitudes and hesitancy toward COVID-19 vaccine", Journal of Dental Education.

8. WHO covid-19 vaccines, https://www.who.int/ emergencies/diseases/novel-coronavirus-

2019/covid-19-vaccines, ngày truy cập 9/5/2021.

\section{ĐộT BIẾN GEN UROPORPHYRINOGEN III COSYNTHASE TRÊN MộT BÉ GẢ́I MẮC BỆNH PORPHYRIA TẠO HỒNG CẦU BẨM SINH TẠI VIỆT NAM}

\section{TÓM TẮT}

Mở đâu: Bệnh Porphyria tạo hộng cầu bẩm sinh (CEP) là bệnh dỉ truyền lặn trên nhiễm sắc thể thường do rối loạn của quá trình chuyển hóa porphyrin gậy thiếu hụt uroporphyrinogenIII cosynthase (UROS) dẫn đến sự tích lũy uroporphyrinogen I trong tủy xương, gan và các mô. Ca lâm sàng: Mô tả đặc điểm lâm sàng và đột biến gen trên một bệnh nhân người Việt đến khám tại Bệnh viện Da Liễu Trung Uơng. Bé gái 5 tuổi có tình trạng da bị nhạy cảm nặng với ánh sáng, cụ thể là các thương tổn bóng nưởc và rậm lông ở

\footnotetext{
*Trường Đại học Y khoa Phạm Ngọc Thạch, TP. Hồ Chí Minh

Chịu trách nhiệm chính: Phạm Hiếu Liêm

Email: drkimdao@pnt.edu.vn

Ngày nhận bài: 3.6.2021

Ngày phản biên khoa họ: 31.7.2021

Ngày duyệt bài: 6.8.2021
}

\section{Đào Hoàng Thiên Kim*, Phạm Hiếu Liêm*}

những vùng phơi bày ánh sáng. Lân đầu tiên chúng tôi mô tả đột biến gen UROS trên môt bênh nhân Đông Nam Á và thực hiện thêm chẩn đoán phân tử để xác định cả những người lành ở thể dị hợp không triệu chứng đang mang mầm đột biến trong gia đình của người bệnh CEP. Để làm rõ cơ sở phân tử đâu tiên của gia đình người Việt này, chúng tôi đã tiến hành nhận dạng UROS đột biến và đo lường hoạt động của uroporphyrinogen III cosynthase trên bệnh nhi CEP. Đột biến sai nghĩa đã được phát hiện là một đột biến thay thế $\mathrm{G}$ thành $\mathrm{T}$ tại nucleotide 11.776 và kết quả là valin bi thay thế thành phenylalanin tại codon 3 của exon 2. Chúng tôi ghi nhận bệnh nhân bị đột biến lặn thể đồng hợp còn bố mẹ của bé ở dạng dị hợp. Hoạt động của đột biến enzym UROS được biểu hiện trong Escherichia coli bị giảm còn $16,1 \%$, chứng tỏ sự giảm rõ rêt hoat đônng của UROS là kết quả chính yếu từ đôt biến gen gây ra bệnh CEP. Kết luận: Phân tích đột biến gen gây bênh CEP được xem là quan trong trong việc quản lý bệnh. Tham vấn di truyền cho toàn bộ 